\title{
Monolithic integration of InP on Si by molten alloy driven selective area epitaxial growth
}

Viazmitinov, Dmitrii V.; Berdnikov, Yury; Kadkhodazadeh, Shima; Dragunova, Anna; Sibirev, Nickolay; Kryzhanovskaya, Natalia; Radko, llya; Huck, Alexander; Yvind, Kresten; Semenova, Elizaveta

\section{Published in:}

Nanoscale

Link to article, DOI:

10.1039/D0NR05779G

Publication date:

2020

Document Version

Peer reviewed version

Link back to DTU Orbit

Citation (APA):

Viazmitinov, D. V., Berdnikov, Y., Kadkhodazadeh, S., Dragunova, A., Sibirev, N., Kryzhanovskaya, N., Radko, I., Huck, A., Yvind, K., \& Semenova, E. (2020). Monolithic integration of InP on Si by molten alloy driven selective area epitaxial growth. Nanoscale, 12(46), 23780-23788. https://doi.org/10.1039/D0NR05779G

\section{General rights}

Copyright and moral rights for the publications made accessible in the public portal are retained by the authors and/or other copyright owners and it is a condition of accessing publications that users recognise and abide by the legal requirements associated with these rights.

- Users may download and print one copy of any publication from the public portal for the purpose of private study or research.

- You may not further distribute the material or use it for any profit-making activity or commercial gain

- You may freely distribute the URL identifying the publication in the public portal 


\title{
Monolithic integration of InP on Si by molten alloy driven selective area epitaxial growth
}

Received 00th January 20xx, Accepted 00th January 20xx DOI: $10.1039 / \times 0 \times x 00000 x$
Dmitrii V. Viazmitinov ${ }^{a, f}$, Yury Berdnikov ${ }^{b, f}$, Shima Kadkhodazadeh ${ }^{c, f}$, Anna Dragunova $^{d}$, Nickolay Sibirev $^{b}$, Natalia Kryzhanovskaya ${ }^{d}$, Ilya Radko ${ }^{e}$, Alexander Huck ${ }^{e}$, Kresten Yvind ${ }^{a, f}$ and Elizaveta Semenova $*^{\mathrm{a}, \mathrm{f}}$

\begin{abstract}
We report a new approach for monolithic integration of III-V materials into silicon, based on selective area growth and driven by a molten alloy in metal-organic vapor epitaxy. Our method includes elements of both selective area and droplet-mediated growths and combines the advantages of the two techniques. Using this approach, we obtain organized arrays of high crystalline quality InP insertions into (100) oriented Si substrates. Our detailed structural, morphological and optical studies reveal the conditions leading to defect formation. These conditions are then eliminated to optimize the process for obtaining dislocation-free InP nanostructures grown directly on Si and buried below the top surface. The PL signal from these structures exhibits a narrow peak at the InP bandgap energy. The fundamental aspects of the growth are studied by modeling the InP nucleation process. The model is fitted by our x-ray diffraction measurements and correlates well with the results of our transmission electron microscopy and optical investigations. Our method constitutes a new approach for the monolithic integration of active III-V material into Si platform and opens up new opportunities in active Si photonics.
\end{abstract}

\section{Introduction}

In virtue of indisputable advantages, such as robustness, sustainable oxide and wide prevalence in nature, Si-based photonics and electronics empowered by complementarymetal-oxide-semiconductor (CMOS) technology are being continuously developed with the aim of lowering power consumption, high-speed operation and continual device miniaturization ${ }^{1-3}$. Since the scaling on silicon devices approaches the physical limit, new materials need to be integrated on to the $\mathrm{Si}$ platform, to ensure further developments in electronics and to achieve new functionalities in photonic applications ${ }^{3}$. III-V materials are, for example, required for active photonic and high-speed electronic applications, due to their direct band gap structures and high electron mobility ${ }^{4}$. This makes monolithic integration of III-V materials onto the Si platform very attractive ${ }^{5}$, as it will have a considerable impact on both research and the industrial production of novel integrated photonics ${ }^{6-9}$, optical interconnects $^{10}$ and high-speed electronic devices ${ }^{11,12}$. Such an

a. DTU Fotonik, Technical University of Denmark, Kongens Lyngby DK-2800, Denmark.E-mail:esem@fotonik.dtu.dk

b. ITMO University, Kronverkskiy 49, 197101 St. Petersburg, Russia.

c. DTU Nanolab-National Centre for Nano Fabrication and Characterization,

Technical University of Denmark, Kongens Lyngby DK-2800, Denmark.

d. National Research University Higher School of Economics, 16 Soyuza

Pechatnikov, St Petersburg 190008, Russia.

e. Center for Macroscopic Quantum States (bigQ), Department of Physics, Technical University of Denmark, , Kongens Lyngby DK-2800, Denmark.

f. $f$ NanoPhoton-Center for Nanophotonics, Technical University of Denmark, DK-

2800 Kongens Lyngby, Denmark

₹ These authors contributed equally.

Electronic Supplementary Information (ESI) available: Additional SEM, HR TEM, PL, EDX and XRD data, the details of the mask preparation, epitaxial growth characterization methods and growth modeling are provided. integration, however, is challenging, due to the large lattice mismatch between Si and III-V materials, their highly dissimilar thermal expansion coefficients and polar-on-nonpolar epitaxy problems, which typically lead to structures with defect density levels above what is acceptable for device fabrication ${ }^{13}$.

In recent years tremendous effort has been devoted to implementing techniques that reduce the number of defects in the III-V nanostructures monolithically integrated into Si. They include wafer bonding ${ }^{14-17}$, transfer printing ${ }^{18}$ and direct epitaxial methods of integration ${ }^{13,19}$, such as growth on thick buffer layers ${ }^{20-22}$, various selective-area growth (SAG) methods ${ }^{12,23-27}$ and different approaches utilizing liquid group III metallic droplet as a growth catalyst $28-30$. The latter two families of techniques are more promising, due to their flexible integration into Si-based circuits and avoiding usage of III-V substrates, complicated wafer bonding techniques and difficulties in alignment of III-V elements to the Si-circuit. They have subsequently already been used to obtain high-quality III$V$ structures grown on Si for laser applications 23,31-33. Besides effective dislocation filtering, the SAG approaches have been used to produce highly ordered nanostructure arrays with precise positioning $13,25,34,35$. The use of group III droplets enables the growth of III-V materials on Si with low defect density, due to a small nucleation area and close to thermodynamic equilibrium growth conditions 28,30,36,37.

Here, we present a new CMOS compatible epitaxial growth technique by metal-organic vapor phase epitaxy (MOVPE), named molten alloy driven selective-area growth (MADSAG). It is based on using a droplet of the group III element of the final crystal combined with the advantages of SAG. Methods of direct selective area epitaxy on silicon usually suffer from crystal 
defects, which cannot be trapped in at least one of the directions ${ }^{13}$, as well as from anti-phase boundaries induced by multinuclear growth ${ }^{38}$. In our method, the molten alloy is used to promote crystallization with a single nucleus for every nanoinsertion.

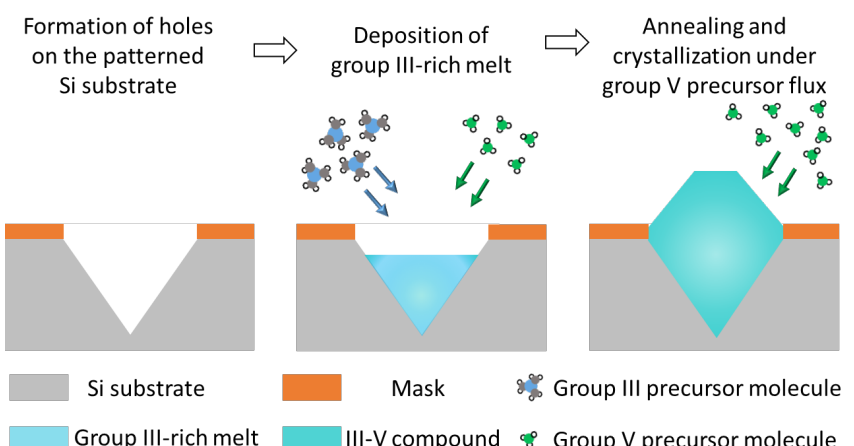

Fig. 1 Schematic diagram for the MADSAG experimental procedure

Implementing this method, we obtain high crystalline quality InP nanoinsertions into (100) oriented Si without using a buffer layer. While our MADSAG method can be used to grow different III-V compounds on $\mathrm{Si}$, the choice of materials here is motivated by further applications in telecom-wavelength compatible photonics ${ }^{39}$, and the otherwise scarce reports of the epitaxy of InP on Si substrates in the literature.

\section{Experimental}

The MADSAG experimental procedure is schematically shown in Fig. 1. The samples investigated here were prepared on Si (100) wafers covered by a $\mathrm{Si}_{3} \mathrm{~N}_{4}$ mask containing arrays of circular openings $200 \mathrm{~nm}$ in diameter and arranged with a pitch of 800 $\mathrm{nm}$. After etching the samples in $\mathrm{KOH}$, inverted pyramidal holes confined by the $\{111\}$ family of planes are formed in each mask opening. The details of the mask fabrication and Si surface preparation are provided in section S1 of the Supplementary Information (SI). The epitaxial growth on the patterned wafers, in turn, consisted of depositing P-rich In melts with subsequent annealing in a $\mathrm{PH}_{3}$ ambient. Details of the epitaxial growth are described in section S2 of the SI. A series of samples were synthesized at different temperatures and $\mathrm{PH}_{3}$ fluxes, in order to understand the InP nucleation by the MADSAG mechanism, through monitoring the evolution of the morphology, chemical composition and crystal structure and quality of the grown nanoinsertions. The structures were studied in detail using scanning electron microscopy (SEM), high resolution scanning transmission electron microscopy (HR STEM), energy-dispersive $\mathrm{X}$-ray spectroscopy (EDX) and X-ray diffractometry (XRD). Their optical properties were investigated using photoluminescence $(\mathrm{PL})$ measurements.

\section{Results and discussion}

We implement our approach to obtain organized arrays of high crystalline quality InP insertions into (100) oriented Si substrates. We present and discuss the results of detailed structural, morphological and optical studies of the obtained structures to identify the growth conditions leading to no defect formation. We identify the impact of the $\mathrm{PH}_{3}$ flux and substrate temperature during the annealing to optimize the process for obtaining dislocation-free $\mathrm{InP}$ nanostructures grown directly on Si and buried below the top surface.

\section{Optimization of the group V flux}

We start by comparing three samples grown identically but annealed under different $\mathrm{PH}_{3}$ fluxes, in order to investigate the impact of the $\mathrm{PH}_{3}$ flux during annealing on the nucleation of $\operatorname{In} \mathrm{P}$ inside the openings in $\mathrm{Si}(100)$. While changing the $\mathrm{PH}_{3}$ flux, the substrate temperature was kept at $600^{\circ} \mathrm{C}$. Moreover, the total number of $\mathrm{PH}_{3}$ molecules per unit surface was kept constant during annealing, by adjusting the annealing time. Images of three samples with the deposited P-rich In melts not annealed, and annealed at $1.2 \times 10^{-2} \mathrm{~mol} / \mathrm{min}\left(15 \mathrm{~min}\right.$ ) and $2.2 \times 10^{-2}$ $\mathrm{mol} / \mathrm{min}$ (8.1 $\mathrm{min}$ ) $\mathrm{PH}_{3}$ flux rates are presented in Fig. 2, respectively. Fig. 2a-c show plan-view SEM images of the structures, Fig. $2 d-f$ show STEM images of the cross-sections of the samples, Fig. $2 \mathrm{~g}-\mathrm{i}$ are fast Fourier transforms (FFT) of the HR STEM images of the regions marked in Fig. $2 d-f$, and Fig. $2 \mathrm{j}-\mathrm{I}$ are EDX composition maps of the samples. Details of the STEM and EDX measurements along with the HR STEM images corresponding to the FFTs in Fig. $2 \mathrm{~g}-\mathrm{i}$ can be found in section S4 of the SI. We find that InP does not nucleate in the sample without annealing, evident by the FFTs of HR STEM images from this sample matching the body-centered tetragonal (bct) crystal structure of In (Fig. 2g). Since the nucleation of the solid phase is known to be limited by the concentration of group $\mathrm{V}$ atoms ${ }^{40,41}$, the $\mathrm{P}$ concentration in this case could not have been high enough to initiate an irreversible crystallization. The concentration of P of $12 \%$ (comparable with the method accuracy), measured by EDX in Fig. $2 \mathrm{j}$, confirms this. Annealing under $a \mathrm{PH}_{3}$ flow is expected to increase the $\mathrm{P}$ concentration in the melt, and subsequently promote the nucleation and further crystallization of InP. Images of the sample annealed at $\mathrm{PH}_{3}$ flux $1.2 \times 10^{-2} \mathrm{~mol} / \mathrm{min}$ suggest a partial nucleation of InP, as regions of both bct In and face centered cubic (fcc) InP can be observed in this sample (see Fig. 2h).

The EDX measurements support this by revealing a nonhomogeneous distribution of $P$ and its segregation in the regions with an $\mathrm{fcc} \operatorname{InP}$ phase (Fig. $2 \mathrm{k}$ ). The $\operatorname{InP}$ region has the same crystallographic orientation as the $\mathrm{Si}$ substrate (see section S4 in the $\mathrm{SI}$ ) and therefore, we conclude that InP nucleates at the interface between the In melt and $\mathrm{Si}$ and it inherits the substrate orientation.

Increasing the $\mathrm{PH}_{3}$ flux leads to increase in the volume of the crystalized InP, evident by the images in Fig. 2c, f, i and I, from the sample annealed at $\mathrm{PH}_{3}$ flux $2.2 \times 10^{-2} \mathrm{~mol} / \mathrm{min}$. Expansion of the volume of material in the nanoinsertions due to complete conversion of In to InP can be clearly seen in the plan-view SEM image in Fig. 2c. HR STEM images and the corresponding FFTs from this sample only show the fcc InP crystal phase, and the $P$ concentration in the EDX map in Fig. 21 has a uniform 
distribution. We observe a sharp interface between the $\operatorname{InP}$ and Si lattices, with the crystal orientation of InP matched to that of the surrounding $\mathrm{Si}$ crystal (see section S4 of $\mathrm{SI}$ ). Further increasing the $\mathrm{PH}_{3}$ flux beyond this value did not considerably affect the outcome.

Based on the results we conclude that the epitaxial growth in the MADSAG process consists of two stages: 1 . deposition of a group III droplet and 2. nucleation and further crystallization of the III-V material during annealing under a group $V$ flux. The nucleation and growth of the InP crystal are, however, driven by the concentration of $\mathrm{P}$ atoms in the In melt. This concentration, in turn, is determined by the difference between the absorption and desorption rates of $\mathrm{P}$ in the In melt. Assuming complete $\mathrm{PH}_{3}$ pyrolysis, we expect that the absorption rate of $\mathrm{P}$ is primarily controlled by the $\mathrm{PH}_{3}$ flux. Thus, when the $\mathrm{PH}_{3}$ flux is kept constant, the $\mathrm{P}$ concentration in liquid In is determined by the substrate temperature, since the desorption of $\mathrm{P}$ is temperature dependent 42
No annealing

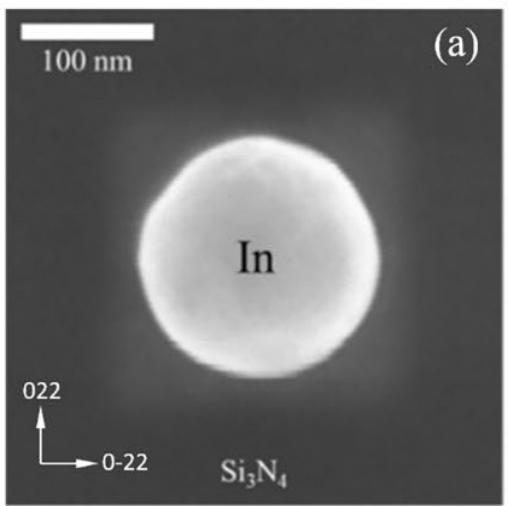

(d)

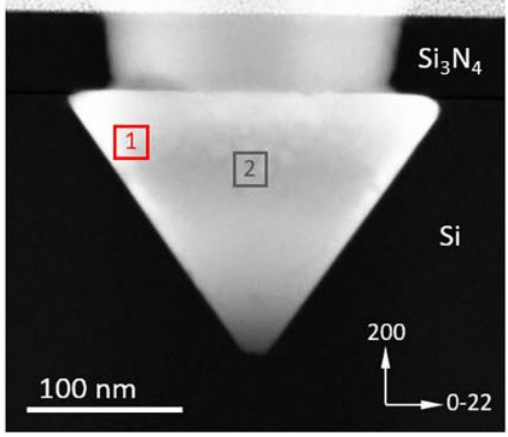

(g)

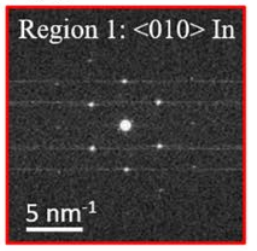

(j) Average $P$ concentration $=12 \%$
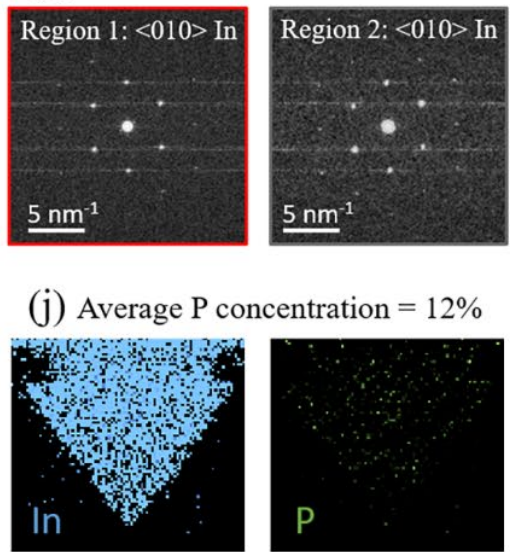

Annealed at $1.2 \times 10^{-2}$ $\mathrm{mol} / \mathrm{min}$
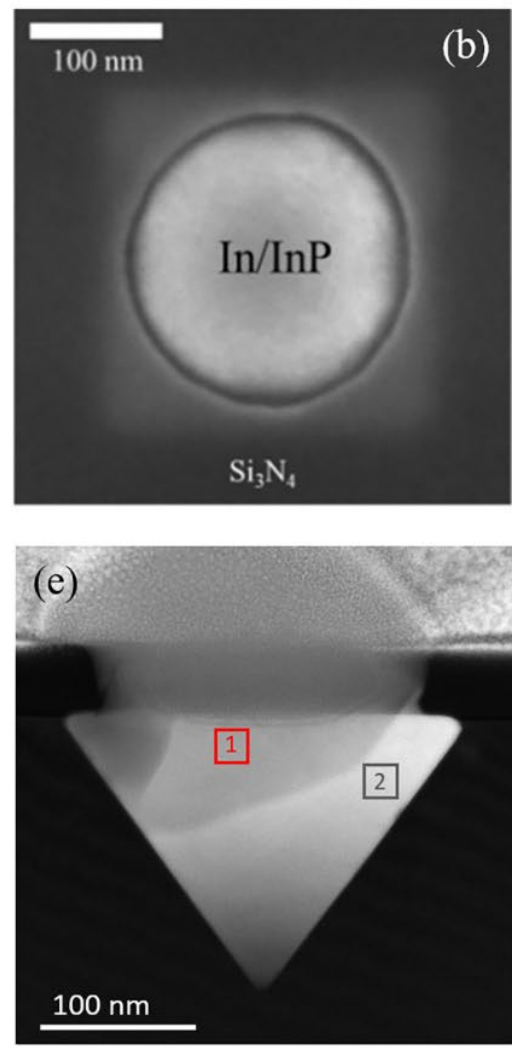

(h)

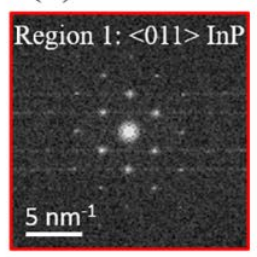

(k) Average $\mathrm{P}$ concentration $=30 \%$
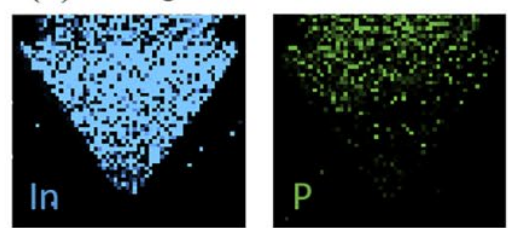

Annealed at $2.2 \times 10^{-2}$ $\mathrm{mol} / \mathrm{min}$

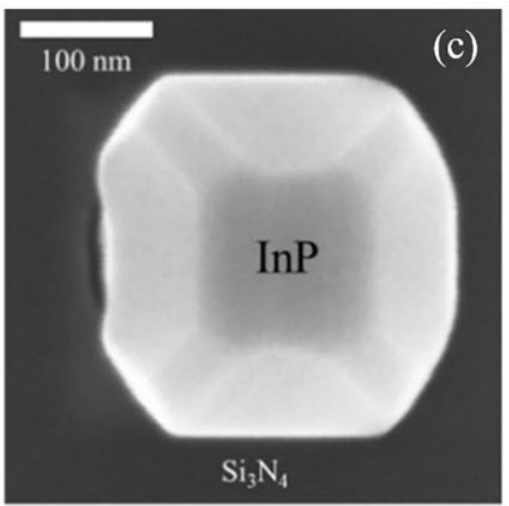

(f)

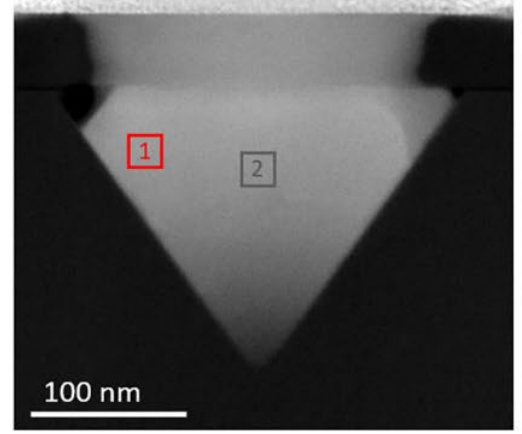

(i)

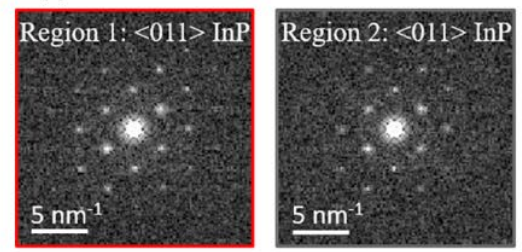

(1) Average $P$ concentration $=47 \%$
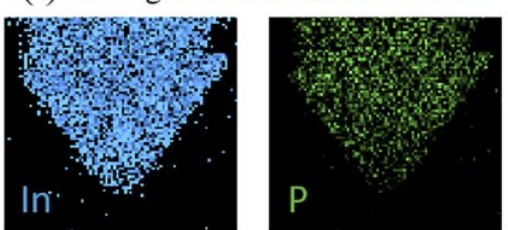

Fig. 2. (a-c) SEM images of nanoinsertion grown in (100) Si and annealed under different conditions in plan-view geometry, (d-f) STEM images of the cross-sections of the nanoinsertions, ( $g-i)$ FFTs of HR STEM images of the regions in the nanoinsertions marked in ( $d-f)$, and $(j-l)$ the corresponding concentration maps of In and $P$, illustrating the crystallization during the MADSAG process without annealing ( $a, d, g, j)$ and with annealing under PH3 flux of $1.2 \times 10-2(b, e, h, k)$ and $2.2 \times 10-2$ mol/min $(c, f, i, l)$. The crystallographic directions of the Si substrate in the plan-view and cross-sectional images are shown in (a) and (d). The EDX measurements have an accuracy of $10-15 \%$. 


\section{ARTICLE}

\section{Optimization of the substrate temperature}

In order to better understand how the annealing temperature affects the MADSAG process, we have investigated the optical properties, morphology and crystalline quality of a set of nanoinsertions annealed at different temperatures under a $\mathrm{PH}_{3}$ flux of $2.2 \times 10^{-2} \mathrm{~mol} / \mathrm{min}$. Fig. 3 compares the $\mathrm{PL}$ spectra acquired at $77 \mathrm{~K}$ and $290 \mathrm{~K}$ from the structures annealed at temperatures 550,600 and $650^{\circ} \mathrm{C}$. Details of the $\mathrm{PL}$ characterization are provided in section S5 of the SI. The PL signal from the three samples demonstrate similar shapes but have significantly different intensities. The highest PL intensity and the narrowest full width at half maximum (FWHM) are observed from the $\mathrm{InP} / \mathrm{Si}$ structures annealed at $600^{\circ} \mathrm{C}$, and the signals from the samples annealed at lower and higher temperatures both have decreased $\mathrm{PL}$ intensities. The signal from the sample annealed at $600{ }^{\circ} \mathrm{C}$ shows a narrow PL peak at the energy corresponding to InP in zincblende crystal phase and a contribution from type-II band alignment ${ }^{43-45}$ (see section S5 of the SI for details). The observed PL peaks show FWHM below $25 \mathrm{meV}$ at $77 \mathrm{~K}$ and $55 \mathrm{meV}$ at $300 \mathrm{~K}$, which is attributed to the high crystallinity of the grown InP structures.

Typical plan-view SEM images of arrays of the nanoinsertions after annealing at 550, 600 and $650^{\circ} \mathrm{C}$ are shown in Fig. 4a-c. We conceive that a square-base truncated pyramid shape of the structures in plan-view implies uniform growth of InP on all four etched $\{111\}$ planes in Si and complete crystallization of In into InP. In the samples annealed at $600{ }^{\circ} \mathrm{C}$ (Fig. 4b) most of the structures have the truncated pyramidal shape. The structures annealed at slightly lower temperature (Fig. 4a) show similar morphology, while annealing at higher temperatures leads to irregularities in morphology (Fig. 4c). We relate the morphology variation to crystal defects and residual In after InP crystallization, which can exist under non-optimized growth conditions. In this case of non-optimal growth conditions, crystal defects tend to nucleate at the interface between the remaining In and InP. The crystallinity of these arrays of $\operatorname{In} P$ nanostructures were further examined by XRD.

Fig. 4d-f compares the XRD InP (111) pole figures acquired from the samples annealed at temperatures 550, 600 and $650^{\circ} \mathrm{C}$, respectively. The peaks crystallographicaly aligned with the Si lattice are labeled with Miller indices, while the peaks arising from twinning in the InP crystal are marked with red arrows. The four-fold symmetry of the peaks from twinned structures is related to four possible symmetries inherited from nucleation on each of the four $\{111\}$ Si planes formed in the mask openings. The samples annealed at different temperatures show very similar peak patterns. (a)

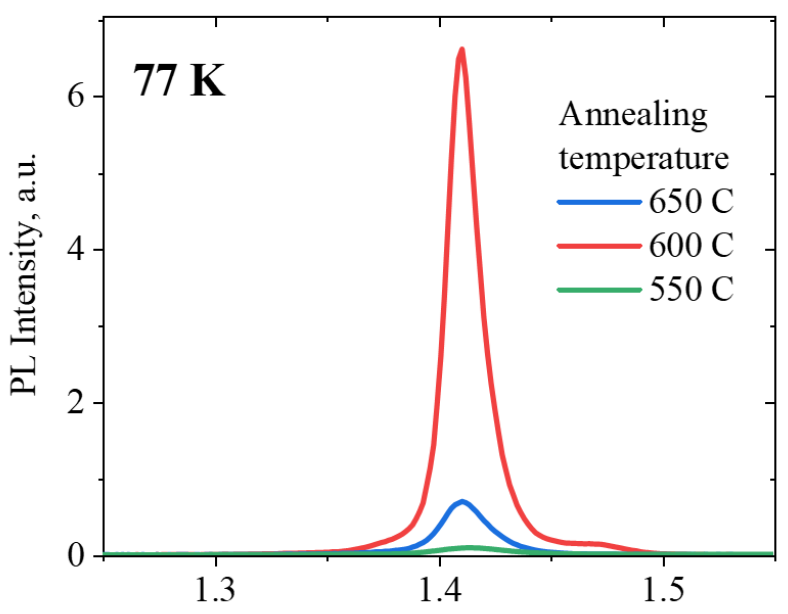

Photon energy, eV

(b)

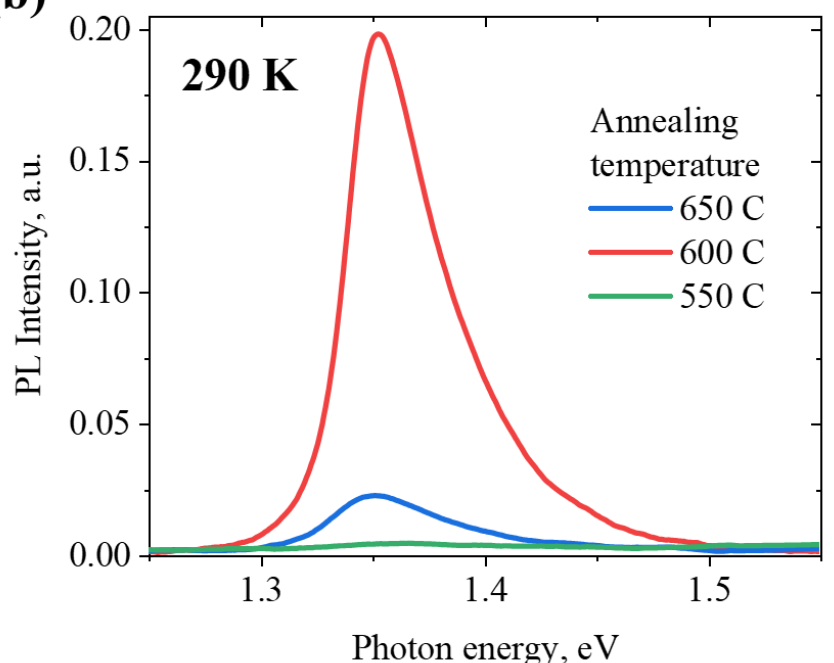

Fig. 3. Photoluminescence spectra acquired at $77 \mathrm{~K}$ (a) and $290 \mathrm{~K}$ (b) from samples annealed at a PH3 flux of $2.2 \times 10-2 \mathrm{~mol} / \mathrm{min}$ and at temperatures 550,600 and $650{ }^{\circ} \mathrm{C}$.

However, the comparison of the integrated intensity of the signal corresponding to the defect-free $\operatorname{InP}\{111\}$ planes, summarized in Table 1, shows that the samples annealed at 550 and $600{ }^{\circ} \mathrm{C}$ have higher intensity of these reflections and thus better crystalline qualities. The comparison of the data with a reference InP wafer can be found in section S6 of the SI.

XRD In (011) pole figures are displayed in Fig. 4g-i, revealing the impact of the annealing temperature on the presence of residual metallic $\mathrm{In}$. The measurements from the three samples demonstrate similar peak positions corresponding to the four $\{110\}$ crystallographic planes, while their intensities vary with 
annealing temperature (integrated reflection intensities are summarized in Table 1).

Table 1. Intensity of $\operatorname{In}(011)$ and $\operatorname{In} P(111)$ reflections

\begin{tabular}{ccc}
\hline $\begin{array}{c}\text { Annealing } \\
\text { temperature, }{ }^{\circ} \mathrm{C}\end{array}$ & $\begin{array}{c}\text { Integral intensity of In } \\
\text { (011) reflection, a. u. }\end{array}$ & $\begin{array}{c}\text { Integral intensity of InP } \\
\text { (111) reflection, a. u. }\end{array}$ \\
\hline 550 & 621 & 16910 \\
600 & 250 & 17002 \\
650 & 1243 & 6842
\end{tabular}

This shows that the residual In in the structures is highly crystalline with a bct crystal structure (space group $14 / \mathrm{mmm}$ ), which is consistent with what we have observed in HR STEM images. Comparing the integral intensity of In (011) reflections in the pole figures from the three samples in Table 1 , we conclude that the sample annealed at $650{ }^{\circ} \mathrm{C}$ has the largest amount of monocrystalline In, whereas the sample annealed at $600^{\circ} \mathrm{C}$ has the least amount of residual In.

The results so far suggest that InP crystals having a symmetrical truncated octahedral pyramid profile in plan-view contain no residual In and are free from defects. Fig. 5 shows images of two structures in cross-sections, one with a such a profile (Fig. 5a-d) and the other with an irregular and asymmetrical plan-view profile (Fig. 5e-h). The EDX maps in Fig. $5 \mathrm{c}$ and $5 \mathrm{~g}$ clearly confirm that the structure with an irregular plan-view shape contains residual In, while the structure with a truncated pyramid shape does not.

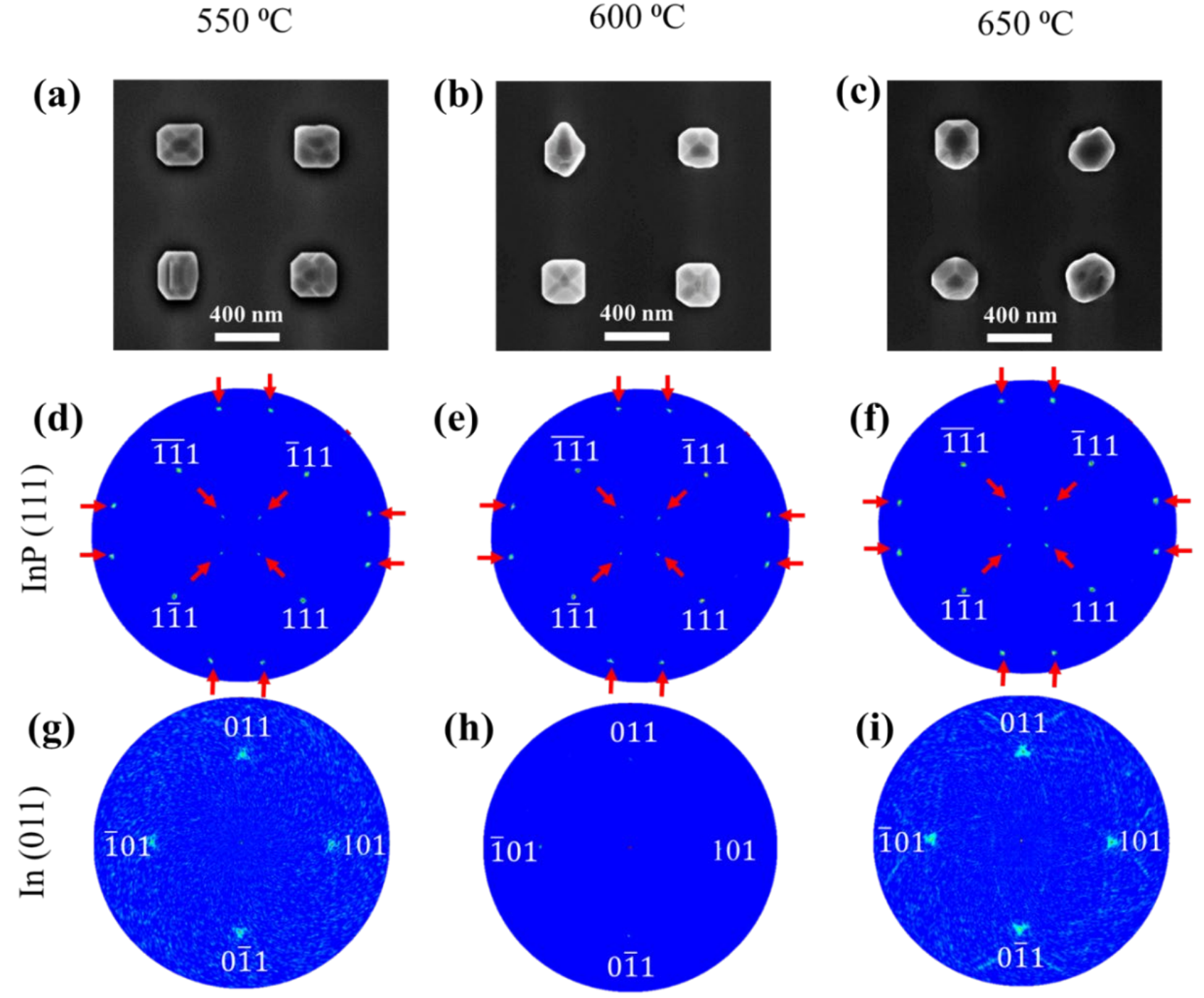

Fig. 4. Top-view SEM images of the InP structures annealed at 550 (a), 600 (b) and $650{ }^{\circ} \mathrm{C}$ (c). InP (111) (d-f) and In (011) (g-i) pole figures acquired from MADSAG InP/Si annealed at 550,600 and $650{ }^{\circ} \mathrm{C}$ respectively. 

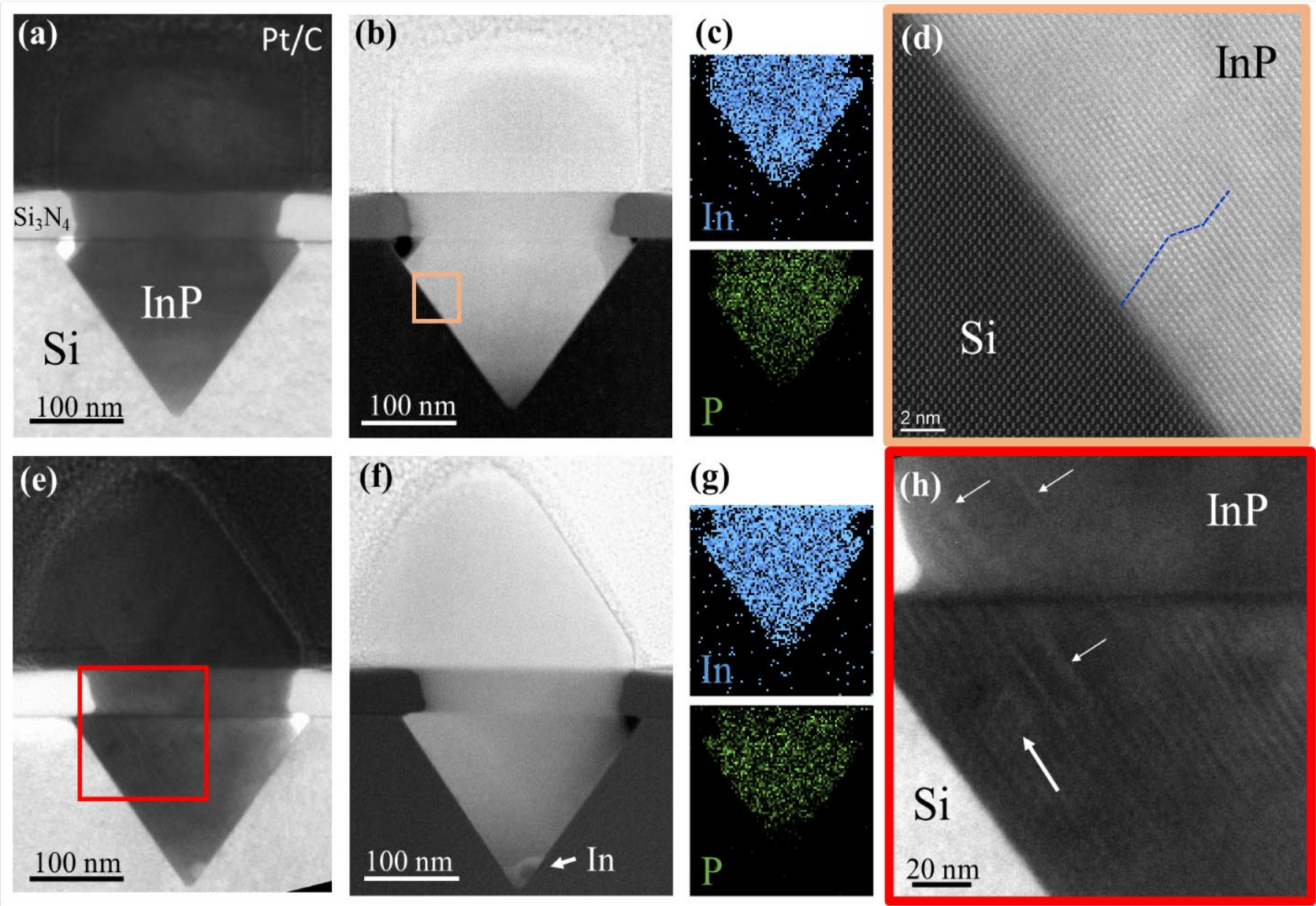

Fig. 5. Bright field TEM images ( $a$, e and h), STEM images ( $b$, $f$ and $d$ ) and EDX maps ( $c$ and $g$ ) comparing InP nanoinsertions with symmetrical ( $a-d)$ and asymmetrical (eh) plan-view profiles. (d) and (h) show zoomed views of the marked regions in (a) and (e), respectively. The dashed blue lines in (d) show a twinning in the InP crystal. The arrows in (h) indicate defects and dislocations in the InP crystal.

Moreover, evidence of dislocations in the nanoinsertions with irregular plan-view profile can be seen in bright field TEM images in Fig. 5 e and h. Dislocations, however, are typically absent in the structures with truncated pyramid plan-view profiles and the only evidence of crystal twinning can be found in these structures, Fig. $5 \mathrm{a}$ and $\mathrm{d}$. Twins are known to have less destructive effect on device performance than other dislocation-type defects, since no dangling bonds form and all atomic bonds are saturated13. However, they typically cause appearance of additional peaks in PL signal obtained from InP nanostructures ${ }^{43-45}$. As we show in section $\mathrm{S} 5$ of the $\mathrm{SI}$, those peaks in our measurements are significantly overlap with the signal from pure zincblende InP crystals. It results in asymmetric widening of cumulative $\mathrm{PL}$ signal and thus defines the abovementioned temperature-dependent FWHM in optimized samples with no remaining In.

We relate the incomplete consumption of In during $\operatorname{In} P$ formation with a lack of $P$ atoms after full coverage of the top surface of the opening by InP crystal, which blocks further transport of $P$ atoms to the In melt. This will be discussed further in the modeling describing the growth process.

We assume that the $\operatorname{In}(011)$ intensity in the XRD data, $I_{I n(011)}$, is directly related to the residual number of In atoms $N_{I n}^{\text {res }}$. We therefore introduce a growth model to estimate the amount of residual In and understand the non-monotonic temperature dependence of the $\ln (011)$ XRD signal.

\section{Growth modeling}

The residual number of In atoms can be calculated as the difference between the initial number of In atoms in melt $N_{\text {In }}$ and the number of In atoms in solid InP. The number of In atoms in InP equals to the number of $P$ atoms, which consist of $P$ dissolved in liquid In before nucleation $N_{P}^{0}$ and absorbed during the crystallization $N_{P}^{\text {cryst }}$, thus:

$N_{I n}^{\text {res }}=N_{I n}-N_{P}^{0}-N_{P}^{\text {cryst }}$.

Nucleation and further crystallization of $\operatorname{In} P$ in the In melt are controlled by $\mathrm{P}$ concentration $C$ driven by the absorption and evaporation of $\mathrm{P}$ atoms. We assume that $C$ is rather uniform inside the In melt, due to the short characteristic time of the species mixing, which can be estimated as $A / D \sim 10^{-5} \mathrm{~s}$, where $A$ is the area of the opening and $D$ is the diffusion coefficient of $\mathrm{P}$ in liquid In, which has the order of magnitude $10^{10} \mathrm{~nm}^{2} / \mathrm{s}^{46}$ at considered growth temperatures.

Before InP nucleation, $\mathrm{P}$ absorption at rate $F$ and evaporation at rate $C / \sigma \tau$ are balanced at the considered growth temperatures $42,47,48$, and thus $C$ is given by the corresponding steady-state value $C_{0}=\sigma F \tau$ and does not depend on time. In our notations, $\sigma$ is the area of the vapor- 
liquid interface per atom, and $\tau$ is the characteristic time of evaporation.

After nucleation, the amount of $\mathrm{P}$ adsorbed during crystallization can be determined by integrating the difference between the absorption and evaporation rates:

$N_{P}^{\text {cryst }}=\int_{0}^{t_{\text {cryst }}}\left(F-\frac{C\left(t^{\prime}\right)}{\sigma \tau}\right) A d t^{\prime}$.

Analytical calculations for $C(t)$ and $t_{\text {cryst }}$ given in the SI allow the determination of $N_{P}^{0}$ and $N_{P}^{\text {cryst }}$ as functions of the annealing temperature $T$. Using the rough estimation of the integral intensity of In (011) reflections $I_{I n(011)} \sim N_{I n}^{\text {res }}$, our modeling result can be summarized as:

$I_{I n(011)}=I_{0}+I_{1} \exp \left[-\frac{T_{v a p}}{T}\right]+I_{2} \exp \left[\frac{T_{v a p}+T_{D}}{T}\right]$

With the temperature-independent term $I_{0}$, and increasing and decreasing exponential functions of the growth temperature with the corresponding prefactors $I_{1}$ and $I_{2}$. The temperaturedependent exponents are controlled by characteristic barriers for P evaporation $T_{\text {vap }}$ and diffusion $T_{D}$ in liquid In. Fig. 6 shows the integral intensity of the pole figure peaks corresponding to $\ln (011)$ reflection fitted by Eq. (3) with fitting parameters $I_{0}=$ 127150, $\mathrm{I}_{1}=228333, \mathrm{I}_{2}=85.7$.

Eq. (3) brings together the effects of several processes, which contribute to the nonmonotonic temperature dependence of the residual amount of In, and thus the intensity of the $\ln (011)$ XRD signal. The increasing exponent $I_{1} \exp \left[-T_{\text {vap }} / T\right]$ in Eq. (3) is proportional to $C_{0}$ and includes two contributions. First, according to our estimations (see section $\mathrm{S} 7$ in the $\mathrm{SI}$ ), the $\mathrm{P}$ concentration before nucleation $C_{0}$ increases with temperature. Thus, less In remains at the end of the process, which corresponds to a decrease of the prefactor $I_{1}$ in Eq. (3) (see section S7 in the SI for details). Second, an increase in $C_{0}$ leads to faster motion of the growth front (shorter $t_{\text {cryst }}$ ). It may lead to the full coverage of the top surface of the opening by $\operatorname{In} P$ before the amount of $P$ required for complete In conversion to InP gets absorbed. Thus, some residual In remains as we show in Fig. 5e, f. A similar situation can occur due to lowering the $\mathrm{PH}_{3}$ flux during the annealing, as shown in Fig. 2c.

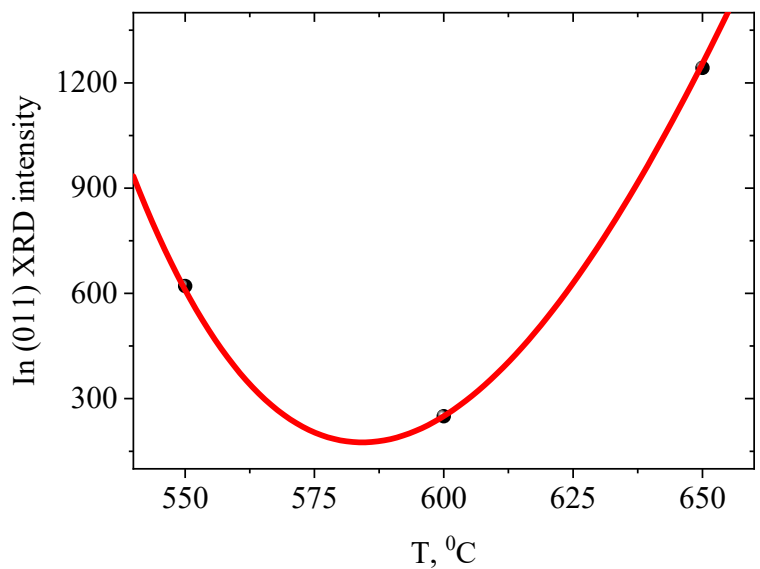

Fig. 6. The integral intensity of $\operatorname{In}(011)$ XRD signal $\mathbf{I}_{\mathbf{I n}(\mathbf{0 1 1})}$ (dots) with the model fit described by Eq. (3) (solid line).
Meanwhile, during a faster crystallization stage less $P$ evaporates from the melt and thus, less In remains, which is represented by the decreasing exponent $I_{2} \exp \left[\left(T_{\text {vap }}+T_{D}\right) / T\right]$ in Eq. (3). Therefore, the competing effects of shorter crystallization time and increasing initial $P$ concentration shape the observed nonmonotonic temperature dependence.

\section{Conclusions}

In conclusion, we have proposed and investigated a new method called MADSAG for the selective-area epitaxial growth of $\mathrm{InP}$ on (100) $\mathrm{Si}$. The InP nanostructures grown under optimized conditions show a very low crystal defect density and sharp interfaces between the grown InP and hosting Si. They also demonstrate a high intensity optical response, verified by PL measurements. We have investigated the influence of the growth parameters on the nucleation and morphology of InP nanoinsertions by SEM, HR STEM, EDX and XRD. Under nonoptimal conditions, residual In remains in the structures, which negatively impacts the crystalline quality and the PL signal of the InP nanoinsertions. The remaining In can be eliminated and complete crystallization of the In melt can be achieved by optimizing the growth temperature and $\mathrm{PH}_{3}$ flux. We thus conclude that MADSAG is a very promising CMOS compatible growth method, which can facilitate the development of novel active hybrid nanodevices integrated into the Si platform.

\section{Conflicts of interest}

There are no conflicts to declare.

\section{Acknowledgements}

The authors acknowledge financial support from Villum Fonden via the NATEC (NAnophotonics for Terabit Communications) Centre (grant no. 8692) and YIP QUEENs (VKR023442) and DNRF Research Centres of Excellence SPOC (ref. DNRF123) and NanoPhoton (ref. DNRF147). YB and NS acknowledge the financial support of growth modeling by the Russian Science Foundation under the grant 19-72-30004. Support of optical measurements from the Basic Research Program of the National Research University Higher School of Economics is gratefully acknowledged.

\section{References}

1 M. Teng, S. Fathpour, R. Safian, L. Zhuang, A. Honardoost, Y. Alahmadi, S. S. Polkoo, K. Kojima, H. Wen, C. K. Renshaw, P. LiKamWa and G. Li, J. Light. Technol., 2020, 38, 6-17.

2 Y. Tanaka, in Metro and Data Center Optical Networks and ShortReach Links III, eds. M. Glick, A. K. Srivastava and Y. Akasaka, SPIE, 2020, p. 19.

3 C. R. Doerr, Front. Phys., 2015, 3, 1-16.

4 J. A. del Alamo, Nature, 2011, 479, 317-323.

5 D.-M. Geum, M.-S. Park, J. Y. Lim, H.-D. Yang, J. D. Song, C. Z. Kim, E. Yoon, S. Kim and W. J. Choi, Sci. Rep., 2016, 6, 20610. 
6 A. W. Elshaari, W. Pernice, K. Srinivasan, O. Benson and V. Zwiller, Nat. Photonics, 2020, 14, 285-298.

7 M. Liao, S. Chen, J.-S. Park, A. Seeds and H. Liu, Semicond. Sci. Technol., 2018, 33, 123002.

8 Y. Yu, W. Xue, E. Semenova, K. Yvind and J. Mork, Nat. Photonics, 2017, 11, 81-84.

9 I. Aharonovich, D. Englund and M. Toth, Nat. Photonics, 2016, 10, 631-641.

10 G. C. Park, W. Xue, M. Piels, D. Zibar, J. Mørk, E. Semenova and I.-S. Chung, Sci. Rep., 2016, 6, 38801.

11 N. Waldron, C. Merckling, L. Teugels, P. Ong, S. A. U. Ibrahim, F. Sebaai, A. Pourghaderi, K. Barla, N. Collaert and A. V.-Y. Thean, IEEE Electron Device Lett., 2014, 35, 1097-1099.

12 M. Borg, H. Schmid, K. E. Moselund, G. Signorello, L. Gignac, J. Bruley, C. Breslin, P. Das Kanungo, P. Werner and H. Riel, Nano Lett., 2014, 14, 1914-1920.

13 B. Kunert, Y. Mols, M. Baryshniskova, N. Waldron, A. Schulze and R. Langer, Semicond. Sci. Technol., 2018, 33, 093002.

14 N. Daix, E. Uccelli, L. Czornomaz, D. Caimi, C. Rossel, M. Sousa, H. Siegwart, C. Marchiori, J. M. Hartmann, K.-T. Shiu, C.-W. Cheng, M. Krishnan, M. Lofaro, M. Kobayashi, D. Sadana and J. Fompeyrine, APL Mater., 2014, 2, 086104.

15 J. Widiez, S. Sollier, T. Baron, M. Martin, G. Gaudin, F. Mazen, F. Madeira, S. Favier, A. Salaun, R. Alcotte, E. Beche, H. Grampeix, C. Veytizou and J.-S. Moulet, Jpn. J. Appl. Phys., 2016, 55, 04EB10.

16 H. K. Sahoo, L. Ottaviano, Y. Zheng, O. Hansen and K. Yvind, J. Vac. Sci. Technol. B, Nanotechnol. Microelectron. Mater. Process. Meas. Phenom., 2018, 36, 011202.

17 A. Sakanas, E. Semenova, L. Ottaviano, J. Mørk and K. Yvind, Microelectron. Eng., 2019, 214, 93-99.

18 A. Osada, Y. Ota, R. Katsumi, M. Kakuda, S. Iwamoto and Y. Arakawa, Phys. Rev. Appl., 2019, 11, 024071.

19 P. Ludewig, S. Reinhard, K. Jandieri, T. Wegele, A. Beyer, L. Tapfer, K. Volz and W. Stolz, J. Cryst. Growth, 2016, 438, 63-69.

20 Y. Wan, J. C. Norman, Y. Tong, M. J. Kennedy, W. He, J. Selvidge, C. Shang, M. Dumont, A. Malik, H. K. Tsang, A. C. Gossard and J. E. Bowers, Laser Photon. Rev., 2020, 2000037.

21 Y. Wan, S. Zhang, J. C. Norman, M. J. Kennedy, W. He, S. Liu, C. Xiang, C. Shang, J.-J. He, A. C. Gossard and J. E. Bowers, Optica, 2019, 6, 1394.

22 I. J. Luxmoore, R. Toro, O. Del Pozo-Zamudio, N. A. Wasley, E. A. Chekhovich, A. M. Sanchez, R. Beanland, A. M. Fox, M. S. Skolnick, H. Y. Liu and A. I. Tartakovskii, Sci. Rep., 2013, 3, 1-5.

23 S. Mauthe, N. Vico Trivino, Y. Baumgartner, M. Sousa, D. Caimi, T. Stoferle, H. Schmid and K. E. Moselund, IEEE J. Sel. Top. Quantum Electron., 2019, 25, 1-7.

24 C. Merckling, N. Waldron, S. Jiang, W. Guo, K. Barla, M. Heyns, N. Collaert, A. Thean and W. Vandervorst, ECS Trans., 2014, 64, 513-521.

25 Z. Wang, B. Tian, M. Pantouvaki, W. Guo, P. Absil, J. Van Campenhout, C. Merckling and D. Van Thourhout, Nat. Photonics, 2015, 9, 837-842.

26 G. Niu, G. Capellini, F. Hatami, A. Di Bartolomeo, T. Niermann, E. H. Hussein, M. A. Schubert, H.-M. Krause, P. Zaumseil, O. Skibitzki, G. Lupina, W. T. Masselink, M. Lehmann, Y.-H. Xie and T. Schroeder, ACS Appl. Mater. Interfaces, 2016, 8, 26948-26955.

27 L. lemmo, A. Di Bartolomeo, F. Giubileo, G. Luongo, M.
Passacantando, G. Niu, F. Hatami, O. Skibitzki and T. Schroeder, Nanotechnology, 2017, 28, 495705.

28 J. Vukajlovic-Plestina, W. Kim, L. Ghisalberti, G. Varnavides, G. Tütüncuoglu, H. Potts, M. Friedl, L. Güniat, W. C. Carter, V. G. Dubrovskii and A. Fontcuberta i Morral, Nat. Commun., 2019, 10, 869.

29 M. Bollani, S. Bietti, C. Frigeri, D. Chrastina, K. Reyes, P. Smereka, J. M. Millunchick, G. M. Vanacore, M. Burghammer, A. Tagliaferri and S. Sanguinetti, Nanotechnology, 2014, 25, 205301. 30 F. Oehler, A. Cattoni, A. Scaccabarozzi, G. Patriarche, F. Glas and J.-C. Harmand, Nano Lett., 2018, 18, 701-708.

31 B. F. Mayer, S. Wirths, S. Mauthe, P. Staudinger, M. Sousa, J. Winiger, H. Schmid and K. E. Moselund, IEEE Photonics Technol. Lett., 2019, 31, 1021-1024.

32 Z. Wang, B. Tian, M. Pantouvaki, W. Guo, P. Absil, J. Van Campenhout, C. Merckling and D. Van Thourhout, Nat. Photonics, 2015, 9, 837-842.

33 J. Herranz, P. Corfdir, E. Luna, U. Jahn, R. B. Lewis, L. Schrottke, J. Lähnemann, A. Tahraoui, A. Trampert, O. Brandt and L. Geelhaar, ACS Appl. Nano Mater., 2020, 3, 165-174.

34 B. Kunert, W. Guo, Y. Mols, B. Tian, Z. Wang, Y. Shi, D. Van Thourhout, M. Pantouvaki, J. Van Campenhout, R. Langer and K. Barla, Appl. Phys. Lett., 2016, 109, 091101.

35 L. Güniat, P. Caroff and A. Fontcuberta i Morral, Chem. Rev., 2019, 119, 8958-8971.

36 T. Tauchnitz, Y. Berdnikov, V. G. Dubrovskii, H. Schneider, M. Helm and E. Dimakis, Nanotechnology, 2018, 29, 504004.

37 M. Pozuelo, H. Zhou, S. Lin, S. A. Lipman, M. S. Goorsky, R. F. Hicks and S. Kodambaka, J. Cryst. Growth, 2011, 329, 6-11.

38 A. M. Raya, M. Friedl, S. Martí-Sánchez, V. G. Dubrovskii, L. Francaviglia, B. Alén, N. Morgan, G. Tütüncüoglu, Q. M. Ramasse, D. Fuster, J. M. Llorens, J. Arbiol and A. Fontcuberta I Morral, Nanoscale, 2020, 12, 815-824.

39 J. Wang, F. Sciarrino, A. Laing and M. G. Thompson, Nat. Photonics, 2020, 14, 273-284.

40 K. Reyes, P. Smereka, D. Nothern, J. M. Millunchick, S. Bietti, C. Somaschini, S. Sanguinetti and C. Frigeri, Phys. Rev. B, 2013, 87, 165406.

41 S. Sanguinetti, S. Bietti and N. Koguchi, in Molecular Beam Epitaxy, Elsevier, 2018, pp. 293-314.

42 K. J. Bachmann and E. Buehler, J. Electrochem. Soc., 1974, 121, 835.

43 K. Li, H. Sun, F. Ren, K. W. Ng, T.-T. D. Tran, R. Chen and C. J. Chang-Hasnain, Nano Lett., 2014, 14, 183-190.

44 A. Zilli, M. De Luca, D. Tedeschi, H. A. Fonseka, A. Miriametro, H. H. Tan, C. Jagadish, M. Capizzi and A. Polimeni, ACS Nano, 2015, 9, 4277-4287.

45 M. H. Hadj Alouane, N. Chauvin, H. Khmissi, K. Naji, B. Ilahi, H. Maaref, G. Patriarche, M. Gendry and C. Bru-Chevallier, Nanotechnology, 2013, 24, 035704.

46 R. Srnánek and P. Hábovčík, J. Cryst. Growth, 1979, 46, 55-58. 47 I. Ansara, C. Chatillon, H. L. Lukas, T. Nishizawa, H. Ohtani, K. Ishida, M. Hillert, B. Sundman, B. B. Argent, A. Watson, T. G. Chart and T. Anderson, Calphad, 1994, 18, 177-222.

48 E. Kuphal, J. Cryst. Growth, 1984, 67, 441-457. 